\title{
Espacialização de sesquióxidos de ferro (Goethita e Hematita) em mancha de solo exposto por meio de sensoriamento remoto hiperespectral
}

\author{
Spatialization of Iron Oxides and Hydroxides (Goethite and Hematite) in Bare Soil Spot by means of Hyperspectral \\ Remote Sensing
}

Charles Dayler Silva de Almeida ${ }^{1}$

Gustavo Macedo de Mello Baptista ${ }^{1}$

Tati de Almeida ${ }^{1}$

\author{
${ }^{1}$ Instituto de Geociências, Universidade de Brasília - UnB. Campus Darcy Ribeiro - Caixa Postal 4465 - $70910-900$ - \\ Asa Norte - Brasília- DF. \\ charles@agroambiental.agr.br,gmbaptista@unb.br,tati_almeida@unb.br
}

Recebido em 10/12/2015 - Aceito em 19/05/2016

Received on 10/12/2015 - Accepted on 19/05/2016

\begin{abstract}
RESUMO - Métodos convencionais para caracterização de solos são caros e demorados, o que abre espaço para novas técnicas, como o sensoriamento remoto. O objetivo do presente trabalho é testar o potencial do sensor Hyperion na espacialização de goethita e hematita em latossolos e testar a interferência de dois corretores atmosféricos nos valores obtidos. As imagens receberam três pré-processamentos: 1) dados brutos; 2) exclusão de bandas ruidosas; e 3) destriping. Após o pré-processamento realizou-se a análise por componentes principais, bem como correção atmosférica pelos métodos QUAC (Quick Atmospheric Correction) e FLAASH (Fast Line-of-sight Atmospheric Analysis of Spectral Hypercubes). Posteriormente foi escolhido um local de estudo para a coleta de amostras georreferenciadas e sua análise de cor pela carta de Munsell. Os dados foram espacializados com uso do Spectral Feature Fitting (SFF), calculando-se o índice RHGtscale para a imagem. O pré-processamento que apresentou o melhor resultado foi a exclusão de bandas ruidosas, servindo de base para os processamentos posteriores. O índice RHGtscale obtido sob a cena corrigida com QUAC apresentou dados coerentes com a variabilidade de cores observada em campo. Novos trabalhos devem ser incentivados para melhor explorar a potencialidade do sensor Hyperion em estudos mineralógicos.
\end{abstract}

Palavras-chave: Sesquióxidos de Ferro, Hyperion, Correção atmosférica, Spectral Feature Fitting.

\begin{abstract}
Conventional methods for the characterization of soils are expensive and time consuming, which makes room for new techniques, such as remote sensing. The aim of this study is to test the potential of Hyperion on the spatial distribution of goethite and hematite in Oxisols and test the interference of two atmospheric correction methods in the obtained values. The images received three preprocessing methods: 1) raw data; 2) excluding noisy bands; and 3) destriping. After preprocessing was carried out by principal component analysis, as well as methods for atmospheric correction with QUAC (Quick Atmospheric Correction) and FLAASH (Fast Line-of-sight Atmospheric Analysis of Spectral Hypercubes). Later, we choose a study site for sample collection and analysis of georeferenced color by the Munsell color chart. The data were spatialized using the Spectral Feature Fitting (SFF), calculating the RHGtscale index for the image. The excluding noisy bands preprocessing method it was the best result, as a basis for further processing. Calculated RHGtscale index the scene corrected with QUAC presented data consistent with the field observed. New works about the Hyperion potentialities were stimulated to better knowledge in the mineralogical studies.
\end{abstract}

Keywords: Iron Oxides and Hydroxides, Hyperion, Atmospheric Correction, Spectral Feature Fitting.

\section{INTRODUÇÃO}

Devido ao potencial agrícola do Brasil, o conhecimento dos tipos de solo e a sua correta espacialização em escalas maiores de trabalho representa fator importante para a tomada de decisões agrárias.
Atualmente os mapeamentos de caráter nacional estão em escalas muito pequenas, a exemplo do projeto RADAM Brasil, cujos dados são apresentados em escalas da ordem de 1:1.000.000, e que representam cerca de $80 \%$ do território nacional. 
O Distrito Federal possui mapeamento pedológico da EMBRAPA CERRADOS, que recobre todo seu território com escala de 1:100.000 (REATTO et al, 2004; FIGUEIREDO et al, 2008).

A obtenção de mapas pedológicos baseando-se em metodologias clássicas acarretam custos elevados, demandam grandes e demoradas campanhas de campo, pessoal altamente qualificado, tornando exequíveis somente em pequenas extensões territoriais (IPPOLITI et al., 2005; FIGUEIREDO et al., 2008).

O sensoriamento remoto e, em especial, o hiperespectral, tem se mostrado alternativa mais rápida e barata que possibilita a obtenção de informações para a caracterização do solo utilizando assinaturas espectrais (BAPTISTA, 2006; BAPTISTA et al., 2011, HUNT et al., 1971; MADEIRA NETTO, 2001; DALMOLIN et al., 2005).

Atualmente, o Hyperion é o único sensor hiperespectral orbital com dados disponíveis. Esse sensor captura 242 bandas contíguas de $10 \mathrm{~nm}$ cada cobrindo a faixa do espectro óptico refletido na região compreendida entre 356 e $2577 \mathrm{~nm}$. Apesar de apresentar 242 bandas, somente 196 bandas são calibradas radiometricamente, e, portanto, podem ser utilizadas nos estudos espectrais. Suas imagens possuem $7,5 \mathrm{~km}$ de comprimento perpendicular à linha de órbita e $42 \mathrm{~km}$ de comprimento como padrão de cena, mas pode atingir $185 \mathrm{~km}$ de extensão. Sua resolução radiométrica é de 12bits e a resolução espacial é de $30 \mathrm{~m}$ (FOLKMAN et al., 2001; PEARLMAN et al., 2001; BECK, R. 2003; GALVÃO et al., 2009; PETROPOULOS et al., 2012).

A caracterização da composição química dos diferentes tipos de solos ferruginosos a partir de feições espectrais ou bandas de absorção tem sua origem nas transições eletrônicas que predominam na região do visível e infravermelho próximo. A curva espectral da goethita apresenta feições de absorção nos comprimentos de onda de 435, 480, 650 e $917 \mathrm{~nm}$ e da hematita, 445, 530,650 e $885 \mathrm{~nm}$, com destaque para as de efeito de campo cristalino $2(6 \mathrm{~A} 1)=>2(4 \mathrm{~T} 1)$ que ocorrem em 480 nm para a goethita e em $530 \mathrm{~nm}$ na hematita. Essas feições são as responsáveis pela diferença de cores desses minerais (SHERMAN; WAITE, 1985).

A compreensão dessa variação mineralógica permite inferir o caráter pedohídrico do ambiente, pois em áreas com maior presença de goethita, que é um hidróxido, ocorre a oscilação do lençol freático que determina um ambiente oxi-redutor do ferro. Já em áreas hematíticas o lençol freático está mais profundo, pois o predomínio de um óxido permite inferir que o ambiente é predominantemente oxidante.

O presente trabalho tem por objetivo testar a potencialidade do sensor orbital Hyperion na espacialização de óxidos e hidróxidos de ferro a partir da classificação pelo Spectral Feature Fitting e a utilização de diferentes métodos de correção atmosférica. A validação do experimento foi realizada em campo.

\section{MATERIAL E MÉTODOS \\ 2.1 Área de estudo}

A região de estudo localiza-se no Estado de Goiás, no Município de Planaltina, aproximadamente $2,5 \mathrm{~km}$ a nordeste da cidade de São Gabriel, entorno do Distrito Federal, conforme demonstrada na Fig.1. A economia do município é baseada na agricultura e criação de gado (IBGE, 2010).

A área de estudo apresenta solo desnudo preparado para o plantio com aproximadamente $1 \mathrm{~km}^{2}$ e altitude média de 1100 metros. A área apresenta histórico de plantio de soja, milho, feijão em sistema de agricultura de precisão. O relevo é plano e os solos da área são classificados como latossolos vermelho-amarelos distróficos com textura argilosa (informações obtidas junto ao proprietário com base nas análises existentes).

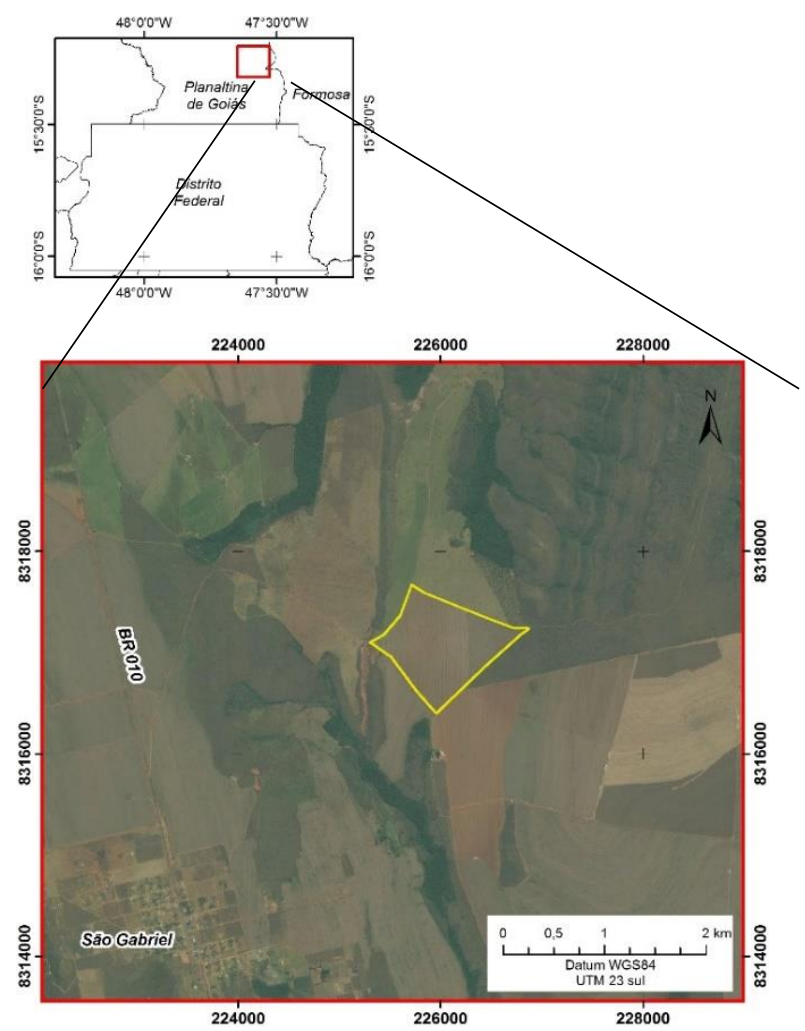

Figura 1 - Localização da área de estudo em amarelo.

\subsection{Aquisição de dados de campo}

A campanha de campo foi realizada entre os dias 21 e 23 de 2011. A metodologia empregada foi a coleta de amostras em transecto ao longo da área para avaliar a cor do solo em relação a tabela de Munsell. As amostras foram coletadas sistematicamente distando 25 metros entre si, totalizando 49 pontos que foram georreferenciados utilizando GPS GARMIN - GPSMAP 62s, com erro médio de $3 \mathrm{~m}$. As coordenadas foram obtidas em UTM, zona 23Sul, datum WGS84.

Os dados coletados, conforme Fig.2, indicam o perfil de solo no ponto A de Latossolo Vermelho distrófico típico que após cerca de 300 metros lineares de distância gradou a um Latossolo Amarelo distrófico típico. A cerca de 600 metros lineares de distância do 
ponto $\mathrm{B}$, término do perfil, novamente prevaleceu o Latossolo Vermelho distrófico típico.

Para a cor do solo foi usada à carta de Munsell, em condições de iluminação natural. As amostras eram umedecidas e então se procedia à classificação para a obtenção do matiz, valor e croma.

Os dados coletados em campo foram utilizados na pesquisa para a validação dos resultados obtidos pelo processamento digital das imagens.

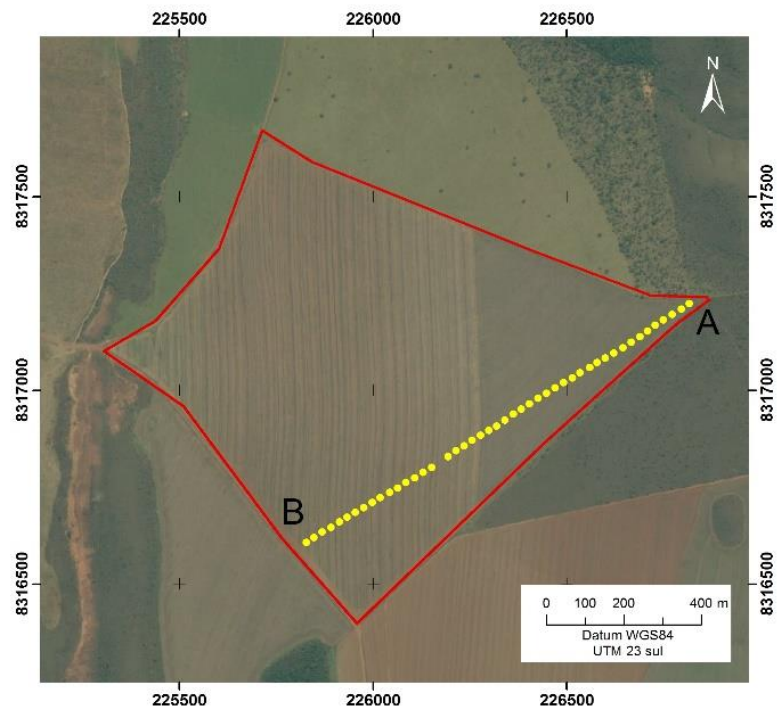

Figura 2: Área de estudo apresentando os pontos de coleta de solo para o transecto A-B.

\subsection{Pré-processamento}

A imagem do sensor Hyperion, obtida no site earthexplorer.usgs.gov, foi adquirida em 08/08/2011. Devido a presença de ruídos, a imagem foi pré-processada para correção radiométrica e atmosférica (Fig.3).

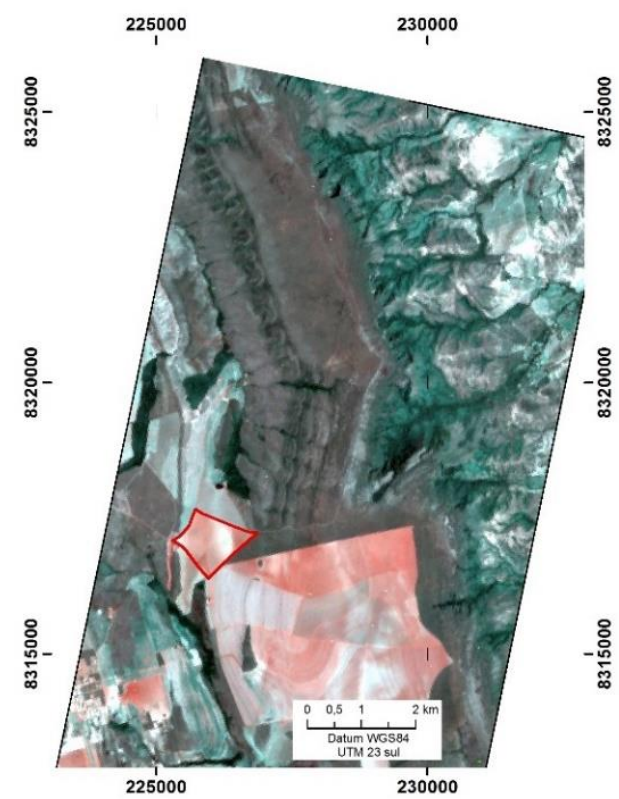

Figura 3: Imagem Hyperion da área de estudo (em vermelho). Composição colorida R: 640nm G: $548 \mathrm{~nm}$ B: $538 \mathrm{~nm}$.
O objetivo dessa etapa foi diminuir a influência do ruído nas imagens Hyperion a fim de melhorar a performance do classificador SFF na área de estudo. $\mathrm{O}$ pré-processamento radiométrico das imagens foi realizado a partir de três métodos distintos (Fig. 4): (i) escolha dos autovetores após a rotação por principais componentes (PCA); (ii) remoção de bandas ruidosas e escolha dos autovetores após a rotação por principais componentes; e (iii) rotina de destripe.

A avaliação dos produtos gerados nos diferentes préprocessamentos foi realizada através das imagens Spectral Feature Fitting dos minerais da goethita e da hematita.

O método Spectral Feature Fitting (SFF) é um ajuste pelo método de mínimos quadrados entre os espectros de reflectância de referência (endmembers) e os pixels das imagens (Baptista, 2006). Esse processamento permite a obtenção de imagens Scale, referentes a concentração dos materiais comparados, e imagens RMS (Root Mean Square) que representa os erros médios quadráticos determinados para cada pixel.

Outro produto desse processamento refere-se a divisão entre a imagem Scale e o RMS e é denominado de Fit Image. Essa imagem representa a probabilidade de o pixel apresentar aquele mineral. $\mathrm{Na}$ imagem gerada pela operação, quanto mais claros os valores dos pixels, que representam os maiores valores, maior será a probabilidade de ocorrência do endmember.

\subsection{Processamento dos dados Hyperion}

Após a remoção dos ruídos das imagens realizada na etapa de pré-processamento e a escolha do método que apresentou os melhores resultados para tal, o índice RHGt (razão Hematita/Goethita) foi obtido utilizando expressão (Equação 1):

$$
\text { RHGtscale }=\frac{\mathrm{Hm}}{\mathrm{Hm}+\mathrm{Gt}}
$$

As variáveis da equação foram as imagens scale do processamento SFF de goethita e hematita, provenientes da correção atmosférica com QUAC e com FLAASH. As imagens scale representam a intensidade das feições de absorção, ou sua profundidade relativa, que segundo Baptista (2001) são proporcionais ao teor quantitativo dos minerais presentes ou apresentado em cada pixel.

Os endmembers utilizados para a determinação do RHGtscale foram obtidos na biblioteca espectral do Jet Propulsion Laboratoty - NASA (JPL-NASA. A escolha desses espectros deve-se ao fato do modelo RHGtscale ter sido desenvolvido utilizando-se essa biblioteca espectral (OLIVEIRA e BAPTISTA, 2001)

A Fig.5 apresenta os espectros da goethita e da hematita reamostrados para as bandas do Hyperion. 


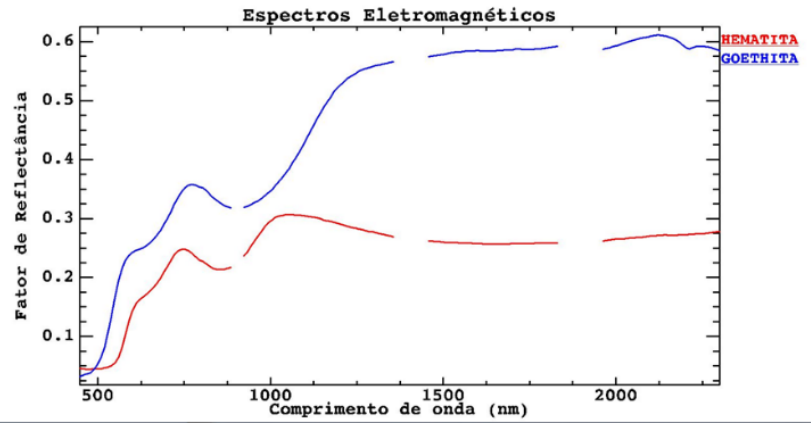

As imagens processadas foram corrigidas geometricamente para a comparação das mesmas com os dados adquiridos na etapa de campo.

Figura 5 - Espectros de Goethita (azul) e Hematita (vermelho) provenientes da biblioteca do JPL-NASA.

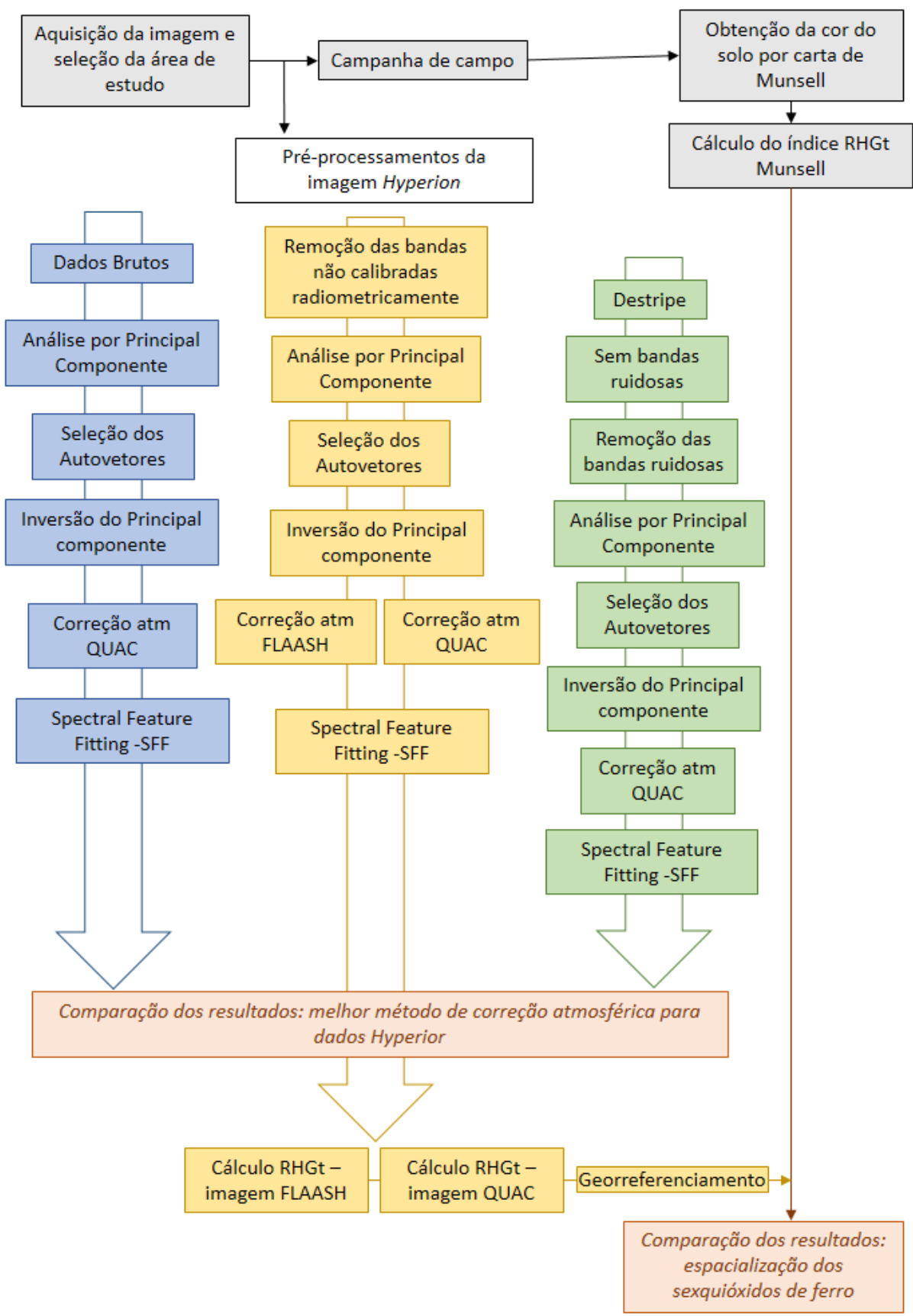

Figura 4-Métodos utilizados na pesquisa 


\section{RESULTADOS E DISCUSSÃO 3.1 Pré-processamento e avaliação}

A avaliação dos métodos de atenuação dos efeitos de stripe nas imagens Hyperion revelou a necessidade de completa remoção das bandas ruidosas para posterior processamento, corroborando com os trabalhos de Goodenough et al. (2003), Tsai et al. (2008) e de Carfantan et al. (2010). Esse fato foi constatado pelo processamento das imagens a partir da classificação SFF (Spectral Feature Fitting) que identificou uma série de inconsistências visuais e estatísticas.

O Spectral Feature Fitting, aplicado nas imagens resultantes da etapa de pré-processamento, gerou diferentes resultados. Os dados provenientes do préprocessamento 3, Vertical Destriping Removal - VDR, quando submetidos ao SFF, apresentaram ruídos na forma de stripes brancas, em colunas centrais, tanto para goethita quanto para hematita, inviabilizando seu uso para o cálculo do índice RHGtscale (Fig.6).

Nas cenas provenientes dos outros dois préprocessamentos o problema verificado nas imagens VDR não foi observado. Contudo, existe diferença na qualidade de exibição dos dados.

A imagem proveniente do processamento 1, ou seja, dados brutos, apresenta uma diferenciação de realce dos dados em relação à imagem. Por isso, optou-se pelos resultados obtidos pelo processamento 2 , com a remoção das bandas não calibradas radiometricamente, adotandose as 10 primeiras ACP (Análise de Componentes Principais) para realizar a remoção dos ruídos.

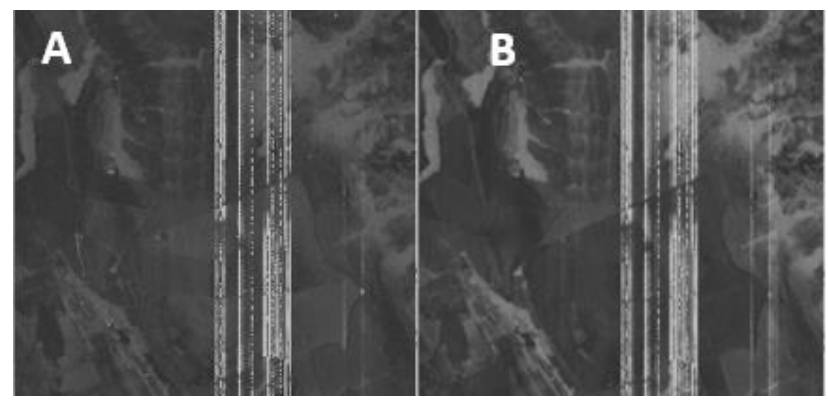

Figura 6 - Imagens pré-processadas pelo método VDR com ruído stripe resultado do processamento SFF. Em (A) imagem Scale Goethita e em (B) Scale Hematita.

A Fig.7 apresenta a comparação entre os processamentos, no qual se percebe melhor contrate para a hematita, obtido na imagem do segundo processamento (Fig. 7B). As setas indicam as regiões que apresentam maiores concentrações de hematita.

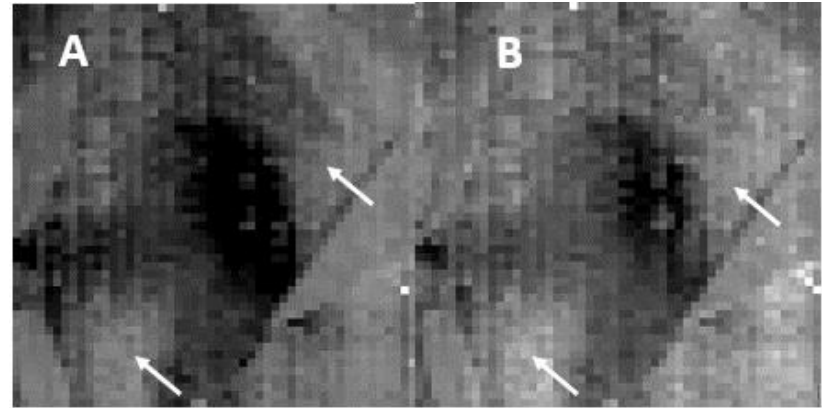

Figura 7 - Comparação entre os dados provenientes do SFF entre os tratamentos 1 (dados brutos - A) e 2 (remoção de banda). As setas indicam as regiões que apresentam maiores concentrações de hematita.

\subsection{Processamento dos dados Hyperion}

Após a definição do pré-processamento para correção radiométria (remoção das bandas ruidosas) e atmosférica (QUAC e FLAASH), adequados aos dados Hyperion, foram obtidos os dados da razão RHGt. Essas imagens de razões dos dados Scale da hematita e da goethita pré-processadas pelo FLAASH e pelo QUACK estão apresentadas na Fig.8.

As imagens foram comparadas com os dados obtidos na etapa de campo utilizando a avaliação de cor baseada na carta de Munsell.

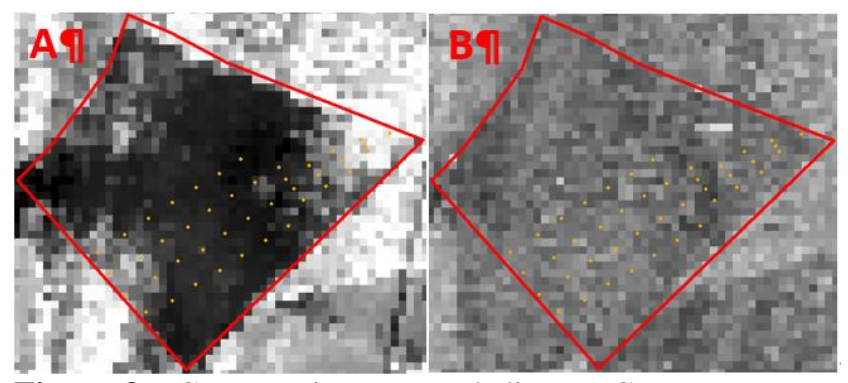

Figura 8 - Comparativo entre o índice RHGt para a imagem QUAC (A) e para o FLAASH (B). Os pontos em cor laranja representam a grade regular aplicada na área de estudo.

Os dados provenientes do QUAC possibilitaram uma boa distinção visual das diferentes concentrações de hematita (áreas claras) das áreas predominantemente goethíticas (áreas escuras), no entanto, na imagem FLAASH essa distinção não foi possível.

Apesar de Agrawal \& Sarup (2011) constatarem uma superioridade nos dados do FLAASH em relação ao QUAC, nesse estudo foi observado que, após a aplicação dos métodos de correção atmosférica e de transformação dos dados de radiância para a reflectância de superfície, os espectros obtidos em ambas as imagens apresentaram feições características das transições eletrônicas do ferro presente no solo. Contudo, ao comparar um pixel de uma porção da cena cujo solo apresenta maior concentração de hematita, percebeu-se que os dados provenientes da correção com o FLAASH apresentaram seu ponto de mínima correspondendo à feição da goethita, enquanto que os dados obtidos por meio do QUAC apresentaram 
seu ponto de mínima correspondendo ao endmember de hematita (Fig.9).

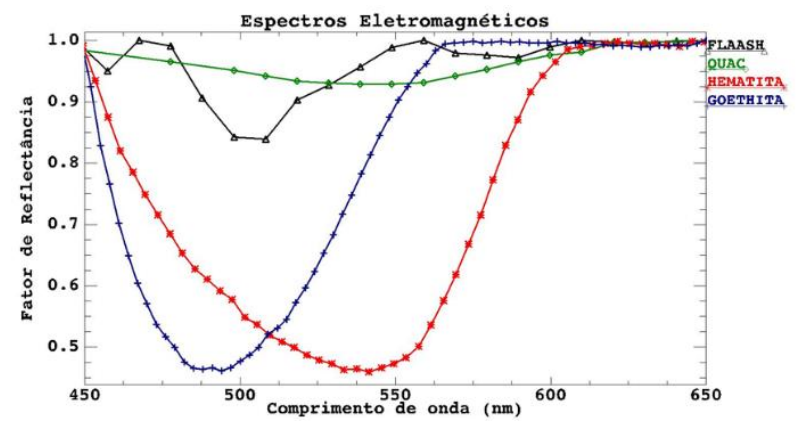

Figura 9 - Comparação entre os endmembers de hematita e de goethita da biblioteca do JPL e os espectros obtidos para um mesmo pixel em dados corrigidos com o FLAASH e com o QUAC. Todos com remoção de contínuo espectral

A partir dos valores digitais dos pixels da imagem foram gerados gráficos para os transectos e para a grade regular (Fig.8). Ao observar o gráfico do transecto A-B, nota-se maiores valores nos pontos iniciais, decrescendo na porção mediana e retomando o crescimento nos pontos finais, situação também encontrada na imagem onde os pontos mais claros da cena QUAC apresentam os maiores valores de concentração de hematita. A Fig.10 apresenta os valores do índice RHGtscale calculados para as cenas corrigidas com o FLAASH e com o QUAC.

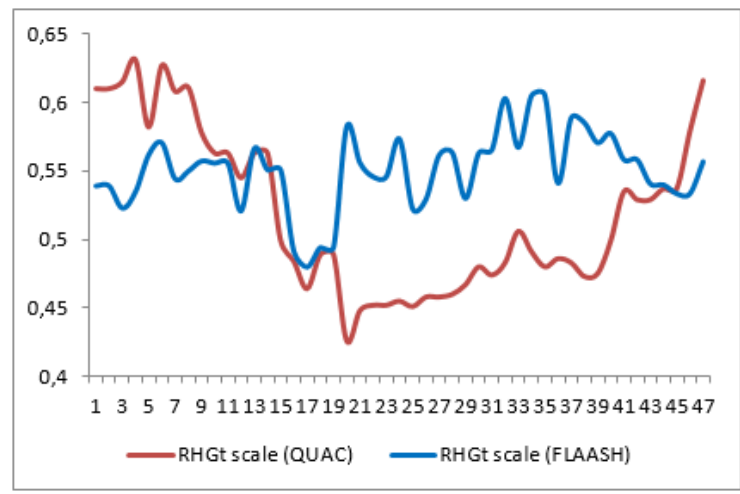

Figura 10 - Valores do índice RHGtscale da imagem para QUAC e FLAASH no transecto A-B.

O transecto inicia-se em uma porção hematítica, passa por uma goethítica e finaliza em outra porção hematítica. $\mathrm{O}$ transecto obtido na imagem RHGtscale por meio dos dados QUAC apresenta-se em concordância com a percepção dos solos no campo. O RHGtscale por meio dos dados FLAASH não apresentou a mesma tendência e tanto na imagem, quanto na análise dos valores dos pixels no transecto, não foi possível fazer distinção das áreas mais hematíticas quanto goethíticas. Tal situação, nos dados corrigidos pelo FLAASH, provavelmente está relacionado ao deslocamento da feição da hematita para a posição ocupada pela feição da goethita na biblioteca espectral, já que o SFF compara os espectros dos pixels da cena com os endmembers.

Os trabalhos de Escadafal (1989) e de Campos (2001) demonstram correlações entre dados de cor e medidas físicas para avaliar discriminação do solo. Ambos enfatizam a necessidade de padronização das observações a fim de ser reduzirem os erros intrínsecos a uma técnica tão subjetiva. Dois fatores importantes destacados são a necessidade de ambiente com iluminação controlada e deve ser realizada por observadores experientes.

Campos (2001) comparou a capacidade de pesquisadores na classificação da cor do solo por meio de índice de precisão das medidas e faz algumas considerações de que somente em $8,75 \%$ das amostras úmidas houve concordância entre a classificação dos pesquisadores e as medidas de colorímetro, observando também que em média os pesquisadores superestimam os valores de matiz.

O fato da determinação da cor ter sido feita em condições de iluminação natural e ter transcorrido ao longo do dia com diferentes condições de iluminação e a inexperiência do avaliador também possuem influências no resultado da coleta dos dados em campo.

Apesar dos indícios visuais de que os dados do FLAASH não apresentam similaridade com o observado em campo, foi realizado um teste " $t$ " no coeficiente B para verificar a correlação entre os dados do transecto A. Em todos os casos testados Munsell x QUAC, Munsell x FLAASH e FLAASH x QUAC não se rejeitou a hipótese nula que $b=0$, ou seja, não há correlação entre as variáveis ou os diversos métodos adotados sob os dados Hyperion.

Com base nos dados que mais se aproximaram da percepção visual no campo, foram geradas as fit images, que podem ser entendidas como a probabilidade de existência do endmember em cada pixel. Na fit image para a goethita (Fig.11) seus altos teores aparecem sob a forma de pixels mais claros e os pixels mais escuros na área de solo exposto correspondem os locais mais ricos em hematita. $\mathrm{O}$ resultado oposto é obtido quando se processa a fit image para a hematita (Fig.12). 


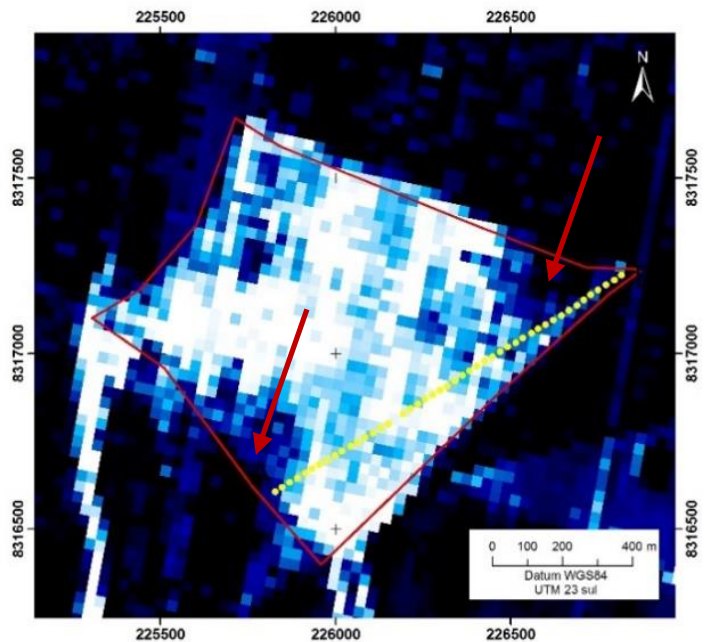

Figura 11 -Fit image de Goethita obtida por meio do processamento SFF. As setas vermelhas indicam áreas com predomínio de porções hematíticas

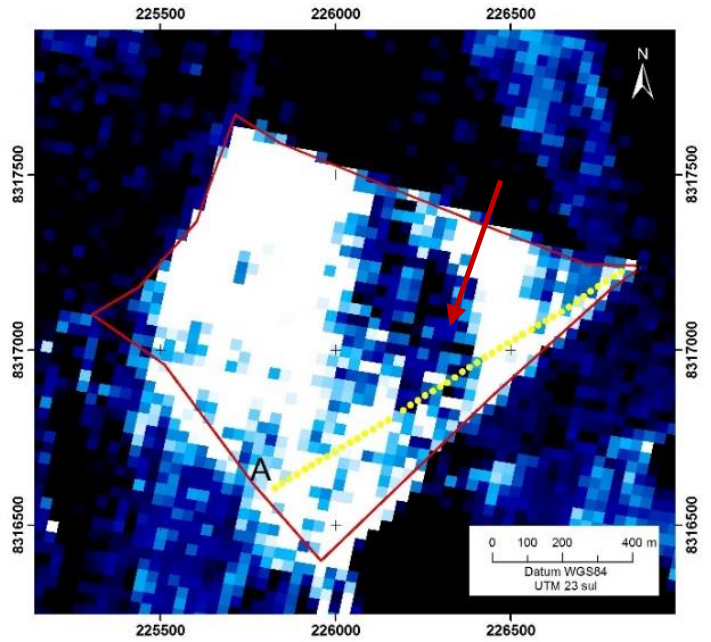

Figura 12 -Fit image de Hematita. A seta vermelha indicam áreas com predomínio de porções goethítica.

Existem locais que apresentam altos valores, bem como baixos valores para os dois minerais, pois eles ocorrem concomitantemente em vários contextos pedológicos (Fig.13). Ou seja, o RHGt scale representou a variabilidade mineralógica dos sesquióxidos de ferro.

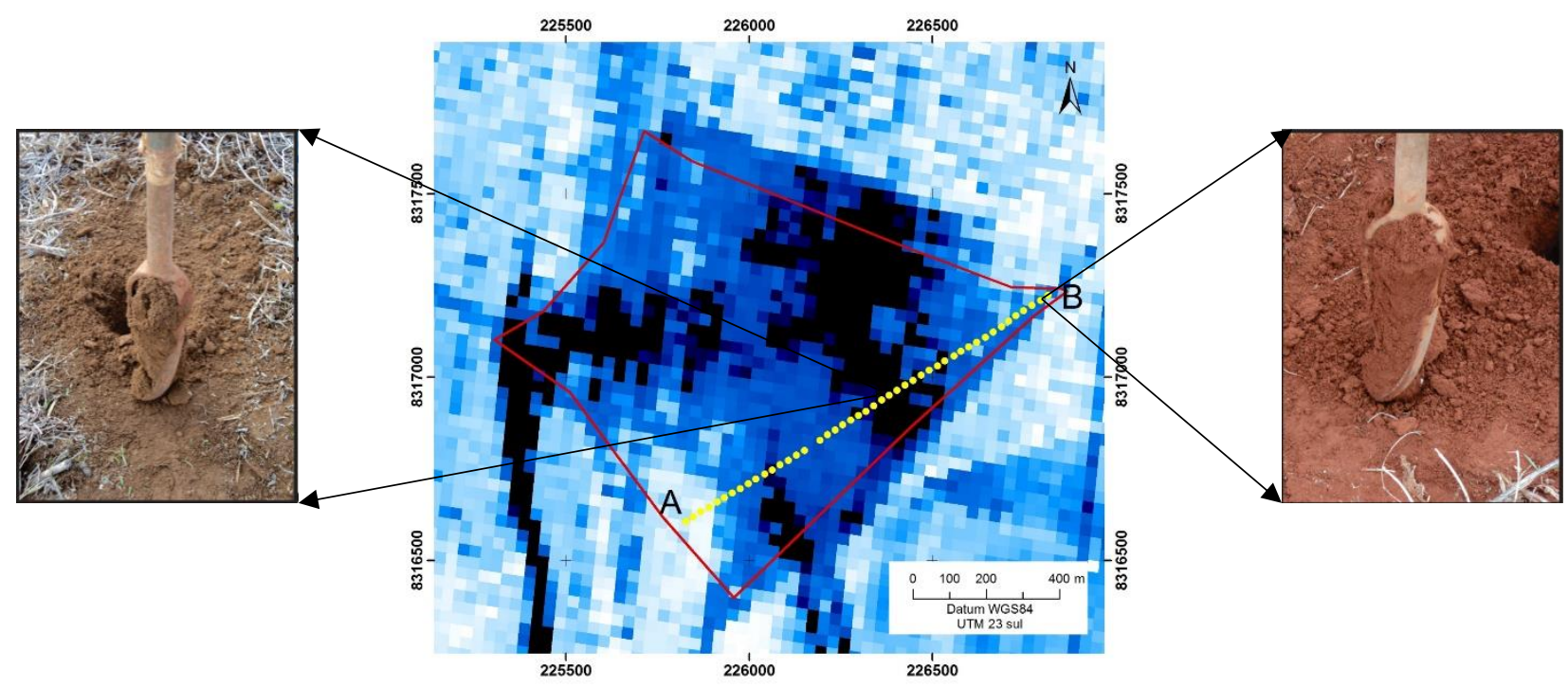

Figura 13 - Imagem ilustrando a cor real dos solos nos locais estudados. Na fotografia superior verifica-se a presença de um latossolo vermelho oriundo da área com maiores teores de hematita e na fotografia inferior solo da região, latossolo amarelo, com dominância de goethita.

\section{4 - CONCLUSÕES E RECOMENDAÇÕES}

No que tange as estratégias de remoção das stripes, o pré-processamento 3, aplicação do Vertical Destriping Removal, foi o que apresentou o melhor resultado. Contudo, no momento em que se aplicou o SFF, surgiram ruídos na forma de stripes brancas por toda a cena, o que inviabilizou a sua utilização para o cálculo do índice RHGtscale. O pré-processamento 2, que consiste de remoção de bandas ruins e considerar as 10 primeiras
ACP Análise por Componentes Principais resultou em dados de boa qualidade viabilizando a sequência dos trabalhos.

A comparação entre os métodos de correção atmosférica mostrou que a espacialização a partir dos dados QUAC estava coerente com as características do solo observado em campo. O mesmo não foi observado ao FLAASH, e há a suspeita de ter sido em função da 
transição eletrônica da hematita estar deslocada em direção à goethita.

$\mathrm{O}$ índice RHGtscale calculado para as imagens possibilitaram a quantificação, contudo só os dados QUAC tinham coerência entre os valores dos pixels e a visualização da imagem, o que também foi mostrado por meio de gráficos.

Para possibilitar a comparação entre os dados de campo artifícios mais eficientes devem ser utilizados para possibilitar a validação dos dados obtidos por imagens hiperespectrais, tais quais, analise de espectro por meio de espectrorradiometros em laboratório, a quantificação dos óxidos e hidróxidos de Fe com ataque sulfúrico, além da difratometria de raios-X.

\section{REFERÊNCIAS BIBLIOGRÁFICAS}

AGRAWAL, G., SARUP, J. Comparision of QUAC and FLAASH Atmospheric Correction Modules on EO-1 Hyperion Data of Sanchi. (Ijaest) International Journal of Advanced Engineering Sciences and Technologies. Vol No. 4, Issue No. 1, 178 - 186, 2011.

BAPTISTA, G. M. M. Sensoriamento Remoto Hiperespetral: o novo paradigma nos estudos de solos tropicais. 1. Ed. Brasília: Editora Universa. v. 1. 2006. $212 \mathrm{p}$.

BAPTISTA, G. M. M.; CORREA, R. S.; SANTOS, P. F.; MADEIRA NETTO, J. S.; MENESES, P. R. Use of Imaging Spectroscopy for Mapping and Quantifying The Weathering Degree of Tropical Soils in Central Brazil. Applied and Environmental Soil Science, v. 2011, p. 1-7, 2011.

BECK, R. EO-1 User Guide - Version 2.3. Department of Geography University of Cincinnati. 2003. 74p.

CAMPOS, R.C. Determinação da cor do solo e sua utilização na predição dos teores de Hematita. Dissertação de mestrado. Escola Superior de Agricultura Luiz de Queiroz, Universidade de São Paulo. Piracicaba - SP. 2001. 59p.

CARFANTAN, H., IDIER, J. Statistical Linear Destriping of Satellite-Based Pushbroom-Type Images. IEEE Transactions On Geoscience And Remote Sensing, VOL. 48, NO. 4, APRIL, 2010.

DALMOLIN, R.S.D., GONÇALVES, C.N., KLAMT, E., DICK, D.P. Relação entre os constituintes do solo e seu comportamento espectral. Ciência Rural, v.35, n.2, mar-abr. 2005.

ESCADAFAL, R., COURAULT, M.C.G.D. Munsell Soft Color and Soil Reflectance in the Visible Spectral Bands of Landsat MSS and TM Data. Remote Sensing of Environment. 27:37-46. 1989.

FIGUEIREDO, S.R., GIASSON, E., TORNQUIST, C.G., NASCIMENTO, P.C. Uso De Regressões Logísticas Múltiplas Para Mapeamento Digital De Solos No Planalto Médio Do Rs. R. Bras. Ci. Solo, 32:2779. 2785, Número Especial. 2008.

FOLKMAN, M., PEARIMAN, J., LIAO, L., JARECKE, P. EO-1/Hyperion hyperspectral imager design, development, characterization, and calibration . Proc. SPIE Vol. 4151. 2001.
GALVÃO, L.S., ROBERTS, D.A., FORMAGGIO, A.R., NUMATA, I., BREUNIG, F.M. View angle effects on the discrimination of soybean varieties and on the relationships between vegetation indices and yield using off-nadir Hyperion data. Remote Sensing of Environment 113, 846-856. 2009.

GOODENOUGH, D.G., DYK, A., NIEMANN, K.O., PEARLMAN, J.S., CHEN, H., HAN, T., MURDOCH, M., WEST, C. Processing Hyperion and ALI for Forest Classification. IEEE Transactions On Geoscience And Remote Sensing, VOL. 41, NO. 6, JUNE. 2003.

HUNT, G.R.; SALISBURY, J.W.; LENHOFF, C.J. Visible and near-infrared spectra of minerals and rocks: III Oxides and hydroxides. Modern Geology, New York, v.1, p.195-205. 1971.

IPPOLITI, G.A.R., COSTA, L.M., SCHAEFER, C.E.G.R., FERNANDES FILHO, E.I., GAGGERO, M.R. Análise Digital Do Terreno: Ferramenta na Identificação de Pedoformas em Microbacia na Região de "Mar de Morros" (MG). R. Bras. Ci. Solo, 29:269-276. 2005.

MADEIRA NETTO, J.S. Comportamento espectral dos solos. In: MENESES, P.R.; MADEIRA NETTO, J.S. Sensoriamento remoto - reflectância dos alvos naturais. Brasília, DF: UnB; Planaltina: EMBRAPA Cerrados. p.127-147. 2001.

OLIVEIRA, R.B.; BAPTISTA, G.M.M. Diferenciação Mineralógica de Solos Lateríticos por meio da Relação Hematita/(Hematita+Goethita), a partir dos Dados do Sensor AVIRIS (Airborne Visible/InfraRed Imaging Spectrometer). CIORD/UnB. Mimeo. 2001.

PETROPOULOS, G.P., ARVANITIS, K., SIGRIMIS, N. Hyperion hyperspectral imagery analysis combined with machine learning classifiers for land use/cover mapping. Expert Systems with Applications, 39, 38003809. 2012.

REATTO, A., MARTINS, É.S., FARIAS, M.F.R., SILVA, A.V., CARVALHO JUNIOR, O.A.C. Mapa pedológico digital - SIG Atualizado do Distrito Federal. Escala 1:100.000 e uma síntese do texto explicativo. Documentos, $n^{\circ} 120$, EMBRAPA CERRADOS, Junho, Planaltina - DF. 2004.

SHERMAN, D.M.; WAITE, T.D. Electronic spectra of $\mathrm{Fe} 3+$ oxides and oxide hydroxides in the near IR to near UV. American Mineralogist. 70, 1262-1269. 1985.

TSAI, F., CHEN, W.W. Striping Noise Detection and Correction of Remote Sensing Images. IEEE Transactions On Geoscience And Remote Sensing, VOL. 46, NO. 12, 2008. 